We are grateful to Journal of Chemical Research for permission to publish the full paper on line.

\title{
Synthesis of novel derivatives of 4-amino-3,5-dicyanopyrazole
}

\author{
M. Sameiro T. Gonçalves ${ }^{\mathrm{a}}$, Ana M.F. Oliveira-Campos ${ }^{* a}$, Lígia M. Rodrigues ${ }^{\mathrm{a}}$, \\ M. Fernanda R.P. Proença ${ }^{a}$, John Griffiths ${ }^{b}$, Hernâni L.S. Maia ${ }^{a}$, Martin Kaja ${ }^{c} \operatorname{and~Radim~Hrdina~}^{c}$ \\ *a Centro de Química, IBQF, Universidade do Minho, Campus de Gualtar, 4710-057 Braga, Portugal \\ ${ }^{\mathrm{b}}$ Department of Colour Chemistry and Dyeing, University of Leeds, Leeds, LS2 9JT, UK \\ ${ }^{\mathrm{c}}$ Department of Organic Technology, Faculty of Chemical Technology, University of Pardubice, Czech \\ Republic
}

Diazotisation of substituted arylamines followed by reaction with malononitrile gave substituted arylazomalononitriles. Cyclisation of these intermediates with chloroacetonitrile and triethylamine, as base, gave the corresponding new aminodicyanopyrazoles, in $22-80 \%$ yields.

Keywords: pyrazole, dicyanopyrazole

Pyrazole derivatives constitute an important family of compounds due to their applications as pharmaceuticals (analgesics, anti-inflammatory, anti-bacterial, andantidepressant), agrochemicals (insecticides) and dyestuffs. ${ }^{1-3}$ Recently aminopyrazoles were found to be potentially useful in preventing protein aggregation which is the first phase of Alzheimer or Creutzfeldt-Jakob diseases. ${ }^{4}$ Pyrazoles are also important synthons for the preparation of biologically active derivatives. ${ }^{5-7}$ The synthesis of these types of heterocyclic rings and related structures has been reviewed. ${ }^{8-10}$

Various methods of synthesis of pyrazoles are known and the most important involves the reaction between hydrazine derivatives with 1,3-difunctional compounds, ${ }^{11}$ such as malononitrile. ${ }^{12}$ To obtain the compounds now described we followed Gewald's method and used arylazomalononitriles as intermediates. Note that arylazomanonitriles have shown a range of interesting biological properties ${ }^{7}$ including anthelmintic, bacteriostatic, ascaricidal and pesticidal.

The arylazomalononitriles may originate from arylpyrazoles through reaction with a two carbon source, such as chloroacetonitrile in basic medium.

It is interesting to recall that 4-amino-5-cyanopyrazoles are not mentioned so often in the literature as their 5-amino-4- cyano analogues. The compounds we synthesised belong to the first type referred to above.

\section{Results and discussion}


The work described here starts by diazotisation of aminobenzoic acids and reaction of the corresponding diazonium salts with malononitrile to give arylazomalononitriles (1). ${ }^{13}$ The intermediates (2) were synthesised by the same method from the adequate starting material (Scheme 1) and were isolated as yellow solids in yields $30-44 \%$.

Previously we reported the successful synthesis of aminocyanopyrazoles containing carboxylic ester groups (3) on the heterocyclic ring. ${ }^{13}$ It was decided to obtain similar heterocyclic compounds containing a nitrile group in place of the ester group $(4,5)$ by the same route. Those would be more versatile to build up directly pyrimidine fused rings containing amino groups. The presence of a reactive ester group such as in compounds $\mathbf{4}$ may allow further reaction with molecules containing amino or hydroxyl groups (such us peptides and sugars).

When the cyclisation of the intermediate 1a was attempted by treatment with chloroacetonitrile in DMF using $\mathrm{K}_{2} \mathrm{CO}_{3}$, the corresponding open chain ester (Scheme 1, structure 2 with $\mathrm{R}=\mathrm{CO}_{2} \mathrm{CH}_{2} \mathrm{CN}$ ) was the only product isolated. ${ }^{13}$ However, when we used triethylamine as the base and chloroacetonitrile both in excess the cyclisation occurred and after purification by column chromatography the 4-amino-3,5-dicyanopyrazoles $\mathbf{4}$ and $\mathbf{5}$ were obtained as solid materials with yields ranging from 22 to $80 \%$ (Scheme 2).

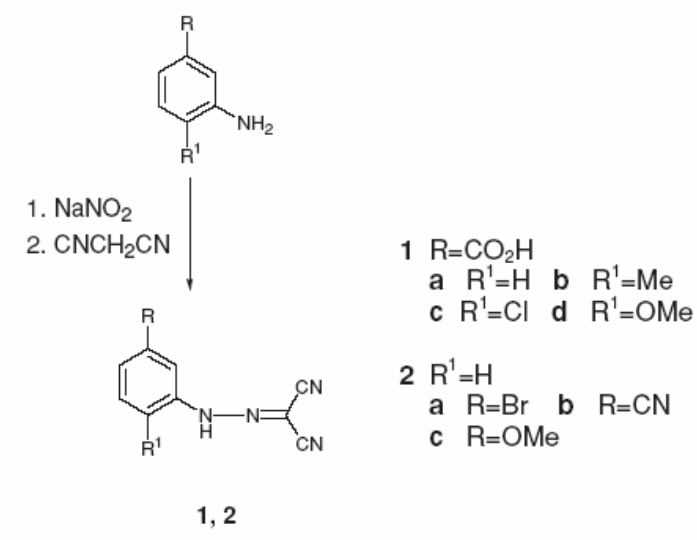

Scheme 1 Synthesis of compounds 1 and 2.

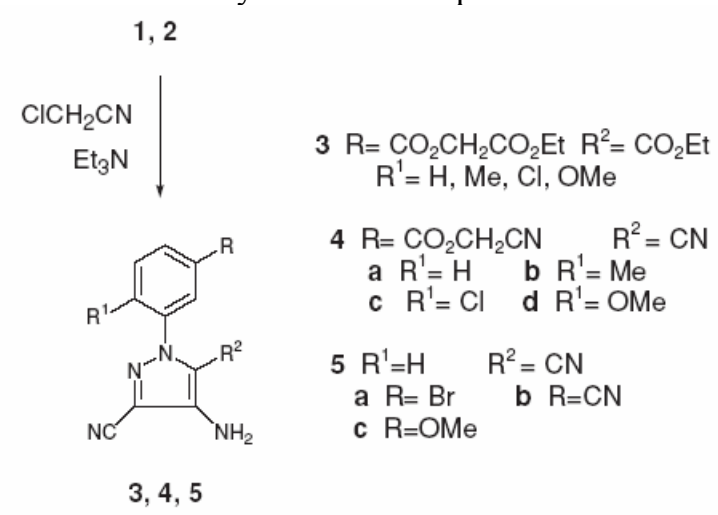

Scheme 2 Synthesis of compounds 3, 4 and 5. 
Two sets of experiments were conducted either heating at reflux for short periods (5-15 minutes, Method A) or in a water bath at $80-90{ }^{\circ} \mathrm{C}$ for longer times (1-4 hours, Method B) (Table 1). In some cases heating for a longer period led to lower yields (4c, $4 \mathbf{d}$ and $\mathbf{5 b}$ ) possibly due to decomposition (tlc) and/or formation of by-products that were not isolated. It is well known that orthoaminonitriles may be very reactive, e.g. towards nitriles. ${ }^{14}$

Table 1 Synthesis of pyrazole derivatives, 4 and 5

\begin{tabular}{|c|c|c|c|c|}
\hline \multirow[b]{2}{*}{ Compound } & \multicolumn{2}{|c|}{ Method A } & \multicolumn{2}{|c|}{ Method B } \\
\hline & Time/min & Yield/\% & Time/hrs & Yield/\% \\
\hline $4 a$ & 5 & 33 & 3 & 80 \\
\hline $4 b$ & 5 & 24 & 4 & 26 \\
\hline $4 c$ & 5 & 52 & 3 & 22 \\
\hline $4 d$ & 10 & 80 & 2 & 25 \\
\hline $5 a$ & 15 & 42 & 4 & 62 \\
\hline $5 b$ & 10 & 70 & 2 & 35 \\
\hline $5 c$ & 15 & 35 & 1 & 65 \\
\hline
\end{tabular}

The formation of pyrazoles $\mathbf{4}$, and 5 was confirmed by their ${ }^{1} \mathrm{H}$ NMR spectra where the signals at highest chemical shift ( $\delta \approx 10-13 \mathrm{ppm}$ ), corresponding to the $\mathrm{N}-\mathrm{H}$ in the intermediates $\mathbf{1}$ and $\mathbf{2}$, were replaced by signals at $\delta \approx 6-7 \mathrm{ppm}$, assigned to the $\mathrm{NH}_{2}$ group of the pyrazoles. The expected pattern for the substituted phenyl ring was observed.

The presence of cyano groups was confirmed by two bands in their IR spectra, e.g.v 2240 and 2224 $\mathrm{cm}^{-1}$ for compound $\mathbf{4 a}$.

In the higher energy region of the IR spectra of all the compounds sharp bands are observed due to $\mathrm{NH}_{2}$ stretching vibrations, e.g. for compound 5c 3662, 3361, $3430 \mathrm{~cm}^{-1}$. For products 4 the ester group is also shown in the IR spectra between 1728 (4d) and $1749(\mathbf{4 b}) \mathrm{cm}^{-1}$.

The ${ }^{13} \mathrm{C}$ NMR data are in agreement with the structures presented. ${ }^{15}$ For compounds $\mathbf{4}$ one signal at $\delta \approx 47.4-47.7 \mathrm{ppm}\left(\mathrm{DMSO}_{-} \mathrm{d}_{6}\right)$ or $57.2\left(\mathbf{4 d}\right.$, acetone- $\left.\mathrm{d}_{6}\right)$ appears, which is attributed to the methylene group from the ester chain. The lowest field signals for compounds $\mathbf{4}$ are due to the carbonyl group. Excluding signals in the aliphatic region, the highest field signals should correspond to carbon atoms 3 and 5 and to cyano carbons.

In the EI mass spectra for compounds $\mathbf{4 a}, \mathbf{b}$ and $\mathbf{d}$ the molecular ion was the base peak. The $\alpha-$ cleavage of the ester group was also observed followed by losses of $\mathrm{CO}$ and $\mathrm{C}_{2} \mathrm{~N}_{2}+\mathrm{H}$, or by the reverse order, probably leading to arylaziridinium ion, as suggested for analogous compounds. ${ }^{16}$ The final compounds were characterised by elemental analysis or high resolution mass spectra.

In conclusion, seven novel compounds were synthesised by a two-step procedure. 
Although the yields were only moderate, it is believed this is a useful contribution since references to 4-amino-5- cyanopyrazoles are scarce.

\section{Experimental}

All melting points were measured on a Gallenkamp apparatus and are uncorrected. IR spectra were determined on a Perkin Elmer FTIR- 1600 using $\mathrm{KBr}$ discs or Nujol emulsions between $\mathrm{NaCl}$ plates. UV spectra were determined on a Hitachi U-2000. NMR spectra were run at $25{ }^{\circ} \mathrm{C}$. ${ }^{1} \mathrm{H} \mathrm{NMR}$ spectra were recorded at $300 \mathrm{MHz}$ and ${ }^{13} \mathrm{C}$ NMR spectra were determined at $75.4 \mathrm{MHz}$ both on a Varian Unity Plus Spectrometer. In the NMR spectra were used $\mathrm{Me}_{4} \mathrm{Si}$ or the solvent peak as internal reference. In ${ }^{1} \mathrm{H}$ NMR spectra all signals mentioned as $\mathrm{d}, \mathrm{t}, \mathrm{dd}$, ddd and $\mathrm{dt}$, are in fact pseudo doublets and triplets.

Low resolution EI mass spectra were determined on a Unicam GCMS120. FAB or high resolution mass spectra were obtained on a VG Ultima or AutoSpec E spectrometers. Elemental analysis data were obtained on a Leco CHNS-932. TLC was carried out on plates coated with $0.25 \mathrm{~mm}$ thick silica gel $60 \mathrm{~F}_{254}$. Column chromatography was performed on silica gel $(<230$ mesh) under conditions that are described below. Light petroleum refers to the fraction boiling in the range $40-$ $60{ }^{\circ} \mathrm{C}$.

General method for synthesising intermediates 1 and 2: To a cooled $\left(0{ }^{\circ} \mathrm{C}\right)$ solution of 3aminobenzoic acid (for intermediates 1) or 3-substituted phenylamine (for intermediates 2$)(3.7 \mathrm{~g}$, $27 \mathrm{mmol})$ in $2 \mathrm{M} \mathrm{HCl}(40 \mathrm{ml})$, a solution of $\mathrm{NaNO}_{2}(1.86 \mathrm{~g}, 27 \mathrm{mmol})$ in water $(7.4 \mathrm{ml})$ was added with stirring. The mixture was kept stirring at RT for 10 minutes and excessive nitrite (the reaction was followed with starch-iodide paper) was destroyed with urea. The resulting diazonium solution was slowly added, with stirring, at low temperature $\left(0^{\circ} \mathrm{C}\right)$ to a solution of malononitrile $(1.78 \mathrm{~g}, 27$ $\mathrm{mmol})$ and $2 \mathrm{M}$ sodium acetate $(20 \mathrm{ml})$ in methanol $(20 \mathrm{ml})$. The mixture was kept stirring for 30 minutes and then left standing for $3 \mathrm{~h}$ at RT. The solid precipitate was filtered, washed with ice water and used in the next step without further purification. Compounds 1a-1d have been described before: ${ }^{13}$

[(3-Bromophenyl)hydrazono]malononitrile (2a): 3-Bromophenylamine was used as starting material. Compound 2a was obtained as a bright yellow solid $(30 \%)$, m.p. $188.4-190.6{ }^{\circ} \mathrm{C} . \delta_{\mathrm{H}}$ $\left(\mathrm{CDCl}_{3}\right) 7.35-7.47(3 \mathrm{H}, \mathrm{m}, 4-\mathrm{H}, 5-\mathrm{H}$ and 6-H), $7.61(1 \mathrm{H}, \mathrm{d} \mathrm{J} 1.5 \mathrm{~Hz}, 2-\mathrm{H}), 12.80(1 \mathrm{H}$, very br s, $\mathrm{NH}$ ). Found: C, 43.36; H, 2.14; N, $22.30 \mathrm{C}_{9} \mathrm{H}_{5} \mathrm{~N}_{4} \mathrm{Br}$ requires $\mathrm{C}, 43.38 ; \mathrm{H}, 2.01 ; \mathrm{N}, 22.49 \%$. 
[(3-Cyanophenyl)hydrazono] malononitrile (2b): 3-Aminobenzonitrile was used as starting material. Compound 2b was obtained as a dark yellow solid (44 \%) m.p. 203.5-205.1 ${ }^{\circ} \mathrm{C}$; $\delta_{\mathrm{H}}$ $\left(\mathrm{CDCl}_{3}+\mathrm{DMSO}_{6}\right)$ 7.40-7.50 (2H, m), $7.76(1 \mathrm{H}, \mathrm{m}, 2-\mathrm{H}), 12.70(1 \mathrm{H}, \mathrm{br} \mathrm{s}, \mathrm{NH})$. Found C, 61.75; $\mathrm{H}, 2.96 ; \mathrm{N}, 35.55 . \mathrm{C}_{10} \mathrm{H}_{5} \mathrm{~N}_{5}$ requires $\mathrm{C}, 61.54 ; \mathrm{H}, 2.58 ; \mathrm{N}, 35.88 \%$.

2-[(3-Methoxyphenyl)-hydrazono]-malononitrile (2c): 3-Methoxyphenylamine was used as starting material to give the compound $2 \mathrm{c}$ as an yellow solid (36\%), it did not melt until $300{ }^{\circ} \mathrm{C}$; $\delta_{\mathrm{H}}$ $\left(\mathrm{DMSO}_{-} \mathrm{d}_{6}\right) 3.76\left(3 \mathrm{H}, \mathrm{s}, \mathrm{OCH}_{3}\right), 6.77(1 \mathrm{H}, \mathrm{dd} \mathrm{J} 8.4$ and $2.4 \mathrm{~Hz}, 4$ or $6-\mathrm{H}), 6.99-7.70(2 \mathrm{H}, \mathrm{m}, 2-\mathrm{H}$ and 4 or 6-H), $7.31(1 \mathrm{H}, \mathrm{t} \mathrm{J} 8.1 \mathrm{~Hz}, 5-\mathrm{H}), 13.00(1 \mathrm{H}$, br s, NH).

General procedure for the cyclisation of compounds 1 and 2: Method A: To the intermediate (1a1d, 2a-2c, $1 \mathrm{mmol})$, chloroacetonitrile $(1 \mathrm{ml})$ and $\mathrm{NEt}_{3}(5 \mathrm{mmol})$ were added with external cooling and the mixture was refluxed for 5-15 minutes (Table 1). After cooling it was poured into water (50 $\mathrm{ml})$ and extracted with ethyl acetate $(4 \times 25 \mathrm{ml})$. The organic extracts were dried $\left(\mathrm{MgSO}_{4}\right)$ and evaporated to dryness giving a brown oil. This product was purified by colunm chromatography.

Method B: To the intermediate $(\mathbf{1 a}-\mathbf{1 d}, \mathbf{2 a}-\mathbf{2 c}, 1 \mathrm{mmol})$ chloroacetonitrile $(1 \mathrm{ml})$ was added and then $\mathrm{NEt}_{3}(5 \mathrm{mmol})$ dropwise with external cooling. The mixture was heated at $80-90{ }^{\circ} \mathrm{C}$ (in a water bath) during 1-4 hours (Table 1). After cooling, water (10-15 ml) was added and a brown oil separated (in the preparation of $\mathbf{5 c}$ this oil solidified as a brown material, and was filtered). The water was decanted and the oil disssolved in ethyl acetate, dried $\left(\mathrm{MgSO}_{4}\right)$ and evaporated to produce a brown oil again. For two cases $(5 \mathbf{a}$ and $\mathbf{5 b}$ ) the oil after rubbing with a $10 \%$ solution of ethyl acetate in ether solidified and the product was filtered and washed with ether giving a brown solid (5a) or an off-white solid (5b). In all other cases the oil was submitted to column chromatography and gave the products as solids.

3-(4-Amino-3,5-dicyanopyrazol-1-yl)-benzoic acid cyanomethyl ester (4a): After column chromatography (ethyl acetate: light petroleum, 1:2) the pyrazole 4a was obtained as a white solid, m.p. 191.6-192.3 ${ }^{\circ} \mathrm{C}$; $v_{\max }(\mathrm{KBr}) 3434,3364,3267,2240,2224,1738,1657,1592 \mathrm{~cm}^{-1} ; \lambda_{\max }$ $(\mathrm{EtOH}) / \mathrm{nm} 225\left(\varepsilon / \mathrm{dm}^{3} \mathrm{~mol}^{-1} \mathrm{~cm}^{-1} 27590\right)$ and $325(8980) \mathrm{nm} ; \delta_{\mathrm{H}}\left(\mathrm{DMSO}_{\mathrm{d}}\right) 5.28\left(2 \mathrm{H}, \mathrm{s}, \mathrm{CH}_{2}\right)$, $6.68\left(2 \mathrm{H}\right.$, br s, $\left.\mathrm{NH}_{2}\right), 7.82(1 \mathrm{H}, \mathrm{t} \mathrm{J} 7.9 \mathrm{~Hz}, 5$ '-H), 8.08 (1H, ddd J 7.9, 2.0 and 1.0 Hz, 6'-H), 8.14 $\left(1 \mathrm{H}\right.$, dt J 7.9 and $\left.1.0 \mathrm{~Hz}, 4^{\prime}-\mathrm{H}\right), 8.27\left(1 \mathrm{H}, \mathrm{t} \mathrm{J} 1.7 \mathrm{~Hz}, 2^{\prime}-\mathrm{H}\right) ; \delta_{\mathrm{C}}\left(\mathrm{DMSO}_{6}\right)$ 161.1, 143.0, 135.4, $128.2,127.8,126.7,125.5,120.8,113.1,111.7,109.6,107.8,96.9,47.4 ; \mathrm{m} / \mathrm{z}(\%)(\mathrm{EI}) 293\left(\mathrm{M}^{+}+1\right.$, 20), $292\left(\mathrm{M}^{+}, 100\right), 236$ (54), 208 (16), 183 (24), 156 (12), 155 (14), 130 (12), 129 (25), 120 (10), 
119 (13), 104 (31), 103 (16), 102 (36), 98 (2), 90 (8), 76 (22), 75 (13). Found: C, 57.80; H, 2.78; N, 28.55. $\mathrm{C}_{14} \mathrm{H}_{8} \mathrm{~N}_{6} \mathrm{O}_{2}$ requires C, 57.53; H, 2.74; N, $28.77 \%$.

3-(4-Amino-3,5-dicyanopyrazol-1-yl)-4-methyl-benzoic acid cyanomethyl ester (4b): After column chromatography (ethyl acetate: light petroleum, 2:3) the pyrazole $\mathbf{4 b}$ was obtained as a yellow solid, m.p. 210.4-212.3 ${ }^{\circ} \mathrm{C}$; $v_{\max }(\mathrm{KBr}) 3431,3362,3261,2237,2226,1749,1654,1587 \mathrm{~cm}^{-1} ; \lambda_{\max }$ $(\mathrm{EtOH}) / \mathrm{nm} 230\left(\varepsilon / \mathrm{dm}^{3} \mathrm{~mol}^{-1} \mathrm{~cm}^{-1} 20000\right)$ and 375 (4900); $\delta_{\mathrm{H}}\left(\mathrm{DMSO}_{6}\right) 2.22(3 \mathrm{H}, \mathrm{s}, \mathrm{Me}), 5.22$ $\left(2 \mathrm{H}, \mathrm{s}, \mathrm{CH}_{2}\right), 6.60\left(2 \mathrm{H}\right.$, br s, $\left.\mathrm{NH}_{2}\right), 7.69\left(1 \mathrm{H}, \mathrm{d} \mathrm{J} 8.0 \mathrm{~Hz}, 5^{\prime}-\mathrm{H}\right), 8.07-8.14\left(1 \mathrm{H}, \mathrm{m}, 4^{\prime}-\mathrm{H}\right), 8.12(1 \mathrm{H}$, s, 2'-H). $\delta_{\mathrm{C}}\left(\right.$ DMSO-d $\left._{6}\right)$ 160.9, 142.0, 139.0, 134.3, 129.9, 128.9, 128.6, 124.4, 111.3, 109.8, 107.5, 98.8, 47.5, 17.7, (the resonance for one carbon, possibly from a cyano group, is not observed); $\mathrm{m} / \mathrm{z}$ (\%) (EI) $307\left(\mathrm{M}^{+}+1,31\right), 306\left(\mathrm{M}^{+}, 100\right), 266$ (9), 254 (20), 253 (34), 250 (53), 227 (39), 226 (33), 222 (18), 221 (42), 169 (63), 142 (21), 116 (26), 115 (21), 90 (20), 89 (40). Found: M $^{+} 306.0847$. Expected for $\mathrm{C}_{15} \mathrm{H}_{10} \mathrm{~N}_{6} \mathrm{O}_{2}: 306.0865$.

3-(4-Amino-3,5-dicyanopyrazol-1-yl)-4-chloro-benzoic acid cyanomethyl ester (4c): After column chromatography (ethyl acetate: light petroleum, 1:1), the pyrazole 4c was obtained as a yellow solid, m.p. $197.2-199.7^{\circ} \mathrm{C}$; $v_{\max }(\mathrm{KBr}) 3439,3358,3248,2975,2246,2222,1739,1644,1581 \mathrm{~cm}^{-1}$; $\lambda_{\max }(\mathrm{EtOH}) / \mathrm{nm} 225\left(\varepsilon / \mathrm{dm}^{3} \mathrm{~mol}^{-1} \mathrm{~cm}^{-1} 25300\right)$ and $315(6450) ; \delta_{\mathrm{H}}\left(\mathrm{DMSO}-\mathrm{d}_{6}\right) 5.22\left(2 \mathrm{H}, \mathrm{s}, \mathrm{CH}_{2}\right)$, $6.65\left(2 \mathrm{H}, \mathrm{s}, \mathrm{NH}_{2}\right), 7.98(1 \mathrm{H}, \mathrm{d}$ J $8.5 \mathrm{~Hz}, 5$ '-H), $8.2(1 \mathrm{H}$, dd J 8.5 and $2 \mathrm{~Hz}, 4$ '-H), 8.38 (1H, d, J 2.0 $\left.\mathrm{Hz}, 2^{\prime}-\mathrm{H}\right) ;$ ); $\delta_{\mathrm{C}}\left(\mathrm{DMSO}_{\mathrm{d}}\right)$ 160.2, 141.9, 133.4, 130.5, 132.6, 129.0, 127.9, 125.8, 113.1, 110.4, 109.5, 107.2, 99.0, 47.7. Found: $\left(\mathrm{M}^{+}+1\right)$ 329.0397. Expected for $\mathrm{C}_{14} \mathrm{H}_{8} \mathrm{~N}_{6} \mathrm{O}_{2}{ }^{37} \mathrm{Cl}: 329.0367$.

3-(4-Amino-3,5-dicyanopyrazol-1-yl)-4-methoxy-benzoic acid cyanomethyl ester (4d): After chromatography $\left(\mathrm{CHCl}_{3}: \mathrm{MeOH}, 100: 3\right)$ the pyrazole 4d was obtained as an beige, m.p. 206.8$208.4^{\circ} \mathrm{C}$; $v_{\max }$ (Nujol) 3438, 3357, 3253, 3217, 2953, 2238, 2227, 1728, 1690, 1647, 1578, 1513 $\mathrm{cm}^{-1} ; \lambda_{\max }(\mathrm{EtOH}) / \mathrm{nm} 235\left(\varepsilon / \mathrm{dm}^{3} \mathrm{~mol}^{-1} \mathrm{~cm}^{-1} 6035\right) 325$ (6064); $\delta_{\mathrm{H}}\left(\mathrm{DMSO}_{6}\right) 3.94\left(3 \mathrm{H}, \mathrm{s}, \mathrm{O} \mathrm{CH}_{3}\right)$, $5.20\left(2 \mathrm{H}, \mathrm{s}, \mathrm{CH}_{2}\right), 6.50\left(2 \mathrm{H}, \mathrm{s}, \mathrm{NH}_{2}\right), 7.49$ (1H, d J $\left.8.0 \mathrm{~Hz}, 5^{\prime}-\mathrm{H}\right), 8.10$ (1H, d J 2.0 Hz, 2'-H), 8.19 $\left(1 \mathrm{H}\right.$, dd J $8.0 \mathrm{~Hz}$ and $\left.2.0 \mathrm{~Hz}, 4^{\prime}-\mathrm{H}\right) . \delta_{\mathrm{C}}\left(\mathrm{CD}_{3} \mathrm{COCD}_{3}\right) 164.2,158.9,144.9,134.6,130.4,127.8,122.1$, 116.0, 115.9, 114.0, 112.5, 110.2, 104.0, 57.2, 50.2; m/z (\%) (EI) $323\left(\mathrm{M}^{+}+1,20\right), 322\left(\mathrm{M}^{+}, 100\right)$, 266 (37), 256 (10), 242 (31), 238 (10), 237 (15), 213 (13), 207 (17), 203 (31), 186 (8), 185 (51), 109 (35), 107 (35), 99 (25), 95 (39), 93 (47), 91 (62), 84 (49), 81 (67), 79 (48). Found: $\mathrm{M}^{+}, 322.0807$. Expected for $\mathrm{C}_{15} \mathrm{H}_{10} \mathrm{~N}_{6} \mathrm{O}_{3}$ : 322.0814. Found: $\mathrm{C}, 55.77 ; \mathrm{H}, 3.20 ; \mathrm{N}, 25.82 . \mathrm{C}_{15} \mathrm{H}_{10} \mathrm{~N}_{6} \mathrm{O}_{3}$ requires $\mathrm{C}$, $55.90 ; \mathrm{H}, 3.11 ; \mathrm{N}, 26.09 \%$. 
4-Amino-1-(3-bromophenyl)-1H-pyrazole-3,5-dicarbonitrile (5a): After filtration (method B) or colunm chromatography (ethyl ether:light petroleum, 3:2; method A) pyrazole 5a was obtained as a brown solid, m.p. $222.5-223.7{ }^{\circ} \mathrm{C} ; \lambda_{\max }(\mathrm{EtOH}) / \mathrm{nm} 206\left(\varepsilon / \mathrm{dm}^{3} \mathrm{~mol}^{-1} \mathrm{~cm}^{-1} 13417\right)$ and $328(6579)$; $v_{\max }$ (Nujol) $3411,3345,3252,2249,2224,1654,1584,1356,1262,789,699 \mathrm{~cm}^{-1} ; \delta_{\mathrm{H}}\left(\mathrm{CD}_{3} \mathrm{COCD}_{3}\right)$ $6.10\left(2 \mathrm{H}\right.$, br s, $\left.\mathrm{NH}_{2}\right), 7.64\left(1 \mathrm{H}, \mathrm{t} \mathrm{J} 8.1 \mathrm{~Hz}, 5^{\prime}-\mathrm{H}\right), 7.77-7.86\left(2 \mathrm{H}, \mathrm{m}, 4^{\prime}\right.$ and 6'-H), 8.00 (1H, t J 2.0 $\left.\mathrm{Hz}, 2^{\prime}-\mathrm{H}\right) ; \delta_{\mathrm{C}}\left(\mathrm{CD}_{3} \mathrm{COCD}_{3}\right) 166.4,146.3,140.1,133.2,132.2,126.5,123.1,122.4,116.1,112.4$, 110.6. Found: $\mathrm{C} 45.81 ; \mathrm{H}, 2.58 ; \mathrm{N}, 23.94 . \mathrm{C}_{11} \mathrm{H}_{6} \mathrm{~N}_{5} \mathrm{Br}$ requires $\mathrm{C}, 45.86 ; \mathrm{H}, 2.10 ; \mathrm{N}, 24.31 \%$.

4-Amino-1-(3-cyanophenyl)-1H-pyrazole-3,5-dicarbonitrile (5b): Filtration (method B) or column chromatography (ethyl acetate: light petroleum, 1:3; method A) afforded the pyrazole $\mathbf{5 b}$ as a light brown solid, m.p 207-208.9 ${ }^{\circ} \mathrm{C} ; \lambda_{\max }(\mathrm{EtOH}, \varepsilon) 213\left(\varepsilon / \mathrm{dm}^{3} \mathrm{~mol}^{-1} \mathrm{~cm}^{-1} 11174\right), 334$ (7969); $v_{\max }$ (Nujol) 3414, 3350, 3254, 2241, 2226, 1565, 1548, 1463, 1378, 1352, 1267, 1020, 891, 795, 722, 689, $670 \mathrm{~cm}^{-1} ; \delta_{\mathrm{H}}\left(\mathrm{CD}_{3} \mathrm{COCD}_{3}\right) 6.16\left(2 \mathrm{H}\right.$, br s, $\left.\mathrm{NH}_{2}\right), 7.92\left(1 \mathrm{H}, \mathrm{t} \mathrm{J} 8.1 \mathrm{~Hz}, 5{ }^{\prime}-\mathrm{H}\right), 8.03(1 \mathrm{H}, \mathrm{d} \mathrm{J} 8.1$ $\mathrm{Hz}, 6$ ' or 4'-H), $8.15\left(1 \mathrm{H}, \mathrm{d} J 8.1 \mathrm{~Hz}, 4^{\prime}\right.$ or 6'-H), $8.23\left(1 \mathrm{H}, \mathrm{t} \mathrm{J} 2.1 \mathrm{~Hz}, 2^{\prime}-\mathrm{H}\right) . \delta_{\mathrm{C}}\left(\mathrm{CD}_{3} \mathrm{COCD}_{3}\right) 139.6$, 134.4 133.8, 132.0, 129.8, 128.0, 127.0, 121.4, 120.1, 114.4, 112.3, 110.6. Found: $\left(\mathrm{M}^{+}+1\right)$ 235.0742. Expected for $\mathrm{C}_{12} \mathrm{H}_{7} \mathrm{~N}_{6}: 235.0732$.

4-Amino-1-(3-methoxyphenyl)-1H-pyrazole-3,5-dicarbonitrile(5c): The pyrazole 5c was obtained, after filtration (method B) or column chromatography (ethyl ether: light petroleum, 3:2; method A) as a bright brown solid m.p. $206.2-208.3^{\circ} \mathrm{C}$. $\lambda_{\max }(\mathrm{EtOH}) 214\left(\varepsilon / \mathrm{dm}^{3} \mathrm{~mol}^{-1} \mathrm{~cm}^{-1} 8379\right)$ and 325 (6114); $v_{\max }$ (Nujol) 3430, 3361, 3262, 2242, 2223, 1658, 1612, 1586, 1500, 1466, 1305, 1282, $1242,859 \mathrm{~cm}^{-1} . \delta_{\mathrm{H}}\left(\mathrm{CD}_{3} \mathrm{COCD}_{3}\right) 3.92\left(3 \mathrm{H}, \mathrm{s}, \mathrm{OCH}_{3}\right), 6.03\left(2 \mathrm{H}\right.$, br s, $\left.\mathrm{NH}_{2}\right), 7.13-7.17\left(1 \mathrm{H}, \mathrm{m}, 4^{\prime}-\right.$ H), 7.32-7.35 (2H, m, 2'-H and 6'-H), $7.55\left(1 \mathrm{H}, \mathrm{t}\right.$ J $\left.8 \mathrm{~Hz}, 5^{\prime}-\mathrm{H}\right)$; $\delta_{\mathrm{C}}\left(\mathrm{CD}_{3} \mathrm{COCD}_{3}\right)$ 161.3, 146.2, 140.1, 131.3, 116.2, 115.5, 112.6, 110.7, 109.2, 56.0. Two signals may be hidden under 116.2 and 115.5 ppm. Found: $\mathrm{M}^{+}$239.0803. Expected for $\mathrm{C}_{12} \mathrm{H}_{9} \mathrm{~N}_{5} \mathrm{O}: 239.0807$.

We thank Junta Nacional de Investigação Científica e Tecnológica (Portugal) for financial support through (IBQFUM) and PRAXIS XXI for support under project PRAXIS/ 2/ 2.1/ QUI/ $44 / 94$ and for a scholarship to M.S.T.Gonçalves (BD-2566-93-RM) and Socrates Programme for Martin Kaja

stay in Portugal. We also thank Miss Elisa Pinto for obtaining the NMR, low resolution MS and elemental analysis data.

\section{References}

1. V. Kepe, F. Požgan, A. Galobič, S. Polanc and M. Kočevar, J. Chem. Soc., Perkin Trans. 1, 1998, 2813 (and references cited therein).

2. P. Cankar, I. Wiedermannova and J. Slouka, Acta Univ. Palacki. Olomuc fac. Rer. Nat. 2002, Chemica, 41, 7. 
3. J. Elguero, Comprehensive Heterocyclic Chemistry, ed. A.R. Katritzky and C.W. Rees, Pergamon Press, Oxford, 1984, vol. 5, p 291.

4. P. Rzepecki, M. Wehner, O. Molt, R. Zadmard, K. Harms and T. Schrader, Synthesis, 2003, 12, 1815.

5. N. Haddad and J. Baron, Tetrahedron Lett., 2002, 43, 2171.

6. W. Holzer and I. Pocher, J. Heterocyclic Chem., 1995, 32, 189.

7. F. Freeman, Synthesis, 1981, 12, 925.

8. J. Elguero, Comprehensive Heterocyclic Chemistry, ed. A.R. Katritzky and C.W. Rees, Pergamon Press, Oxford, 1984, vol. 5, p 273.

9. K. Makino, H.S. Kim and Y. Kurasawa, J. Heterocyclic Chem., 1998, 35, 489.

10. K. Takagi and M. Huber-Habart, J. Heterocyclic Chem., 1996, 33, 1003.

11. A.W. Erian, Chem. Rev., 1993, 93, 1991.

12. K. Gewald, H.J. Jaensch and O. Calderon, E. Ger. Pat., 113 359, 1975.

13. J.C.V.P. Moura, A.M.F. Oliveira-Campos, J. Griffiths, H.L.S. Maia and J.I.N.R. Gomes, J. Chem. Res., 1995, (S), 128, (M), 924 (Part I).

14. E.C. Taylor and A.L. Borror, J. Org. Chem., 1961, 26, 4967.

15. M. Begtrup, G. Boyer, P. Cabildo, C. Cataviela, R.M. Claramunt, J. Elguero, J.I. Gardia and C. Toiron, Magnetic Resonance in Chemistry, 1993, 31, 107.

16. J. Elguero, Comprehensive Heterocyclic Chemistry, ed. A.R. Katritzky and C.W. Rees, Pergamon Press, Oxford, 1984, vol. 5, p. 218. 